\title{
Novel Therapeutic Strategies in the Management of Non-Variceal Upper Gastrointestinal Bleeding
}

\author{
Ari Garber and Sunguk Jang \\ Department of Gastroenterology and Hepatology, Cleveland Clinic Foundation, Cleveland, OH, USA
}

\begin{abstract}
Non-variceal upper gastrointestinal bleeding, the most common etiology of which is peptic ulcer disease, remains a persistent challenge despite a reduction in both its incidence and mortality. Both pharmacologic and endoscopic techniques have been developed to achieve hemostasis, with varying degrees of success. Among the pharmacologic therapies, proton pump inhibitors remain the mainstay of treatment, as they reduce the risk of rebleeding and requirement for recurrent endoscopic evaluation. Tranexamic acid, a derivative of the amino acid lysine, is an antifibrinolytic agent whose role requires further investigation before application. Endoscopically delivered pharmacotherapy, including Hemospray (Cook Medical), EndoClot (EndoClot Plus Inc.), and Ankaferd Blood Stopper (Ankaferd Health Products), in addition to standard epinephrine, show promise in this regard, although their mechanisms of action require further investigation. Non-pharmacologic endoscopic techniques use one of the following two methods to achieve hemostasis: ablation or mechanical tamponade, which may involve using endoscopic clips, cautery, argon plasma coagulation, over-the-scope clipping devices, radiofrequency ablation, and cryotherapy. This review aimed to highlight these novel and fundamental hemostatic strategies and the research supporting their efficacy. Clin Endosc 2016;49:421-424
\end{abstract}

Key Words: Endoscopy; Hemostasis; Peptic ulcer; Embolization, therapeutic; Gastrointestinal hemorrhage

\section{INTRODUCTION}

Non-variceal upper gastrointestinal bleeding (NVUGIB) is defined as gastrointestinal (GI) hemorrhage originating proximal to the ligament of Treitz in the duodenum. While there are myriad sources for NVUGIB (such as gastroduodenal erosions, Mallory-Weiss tears, vascular malformations, and severe esophagitis), peptic ulcer bleeding remains the single most common cause, accounting for $25 \%$ to $67 \%$ of the sources of NVUGIB. ${ }^{1}$ According to the Nationwide Inpatient Sample, the largest inpatient care database in the United States, the incidence of NVUGIB has shown a steady decline over the

Received: July 27, 2016 Revised: September 13, 2016

Accepted: September 13, 2016

Correspondence: Sunguk Jang

Department of Gastroenterology and Hepatology, Cleveland Clinic Foundation, 9500 Euclid Avenue Q3-1, Cleveland, OH 44195, USA

Tel: +1-216-636-5440, Fax: +1-216-444-6284, E-mail: jangs@ccf.org

(cc) This is an Open Access article distributed under the terms of the Creative Commons Attribution Non-Commercial License (http://creativecommons.org/ licenses/by-nc/3.0) which permits unrestricted non-commercial use, distribution, and reproduction in any medium, provided the original work is properly cited. last two decades, from 108 to 78 cases per 100,000. ${ }^{2}$ This trend in the United States appears to be mirrored across the globe. ${ }^{1}$ This decrease in the incidence of NVUGIB has also translated into a decline in the in-hospital mortality rate; the case fatality rate of all non-variceal causes of upper GI bleeding declined from $4.5 \%$ in the late 1980 s to $2.1 \%$ in the 2000 s. $^{2}$ Better understanding of the importance of aggressive hemodynamic resuscitation, prompt initiation of pharmacologic intervention (i.e., proton pump inhibitor [PPI]), and improved efficacy of endoscopic hemostasis have contributed to this positive outcome.

Given the imperative therapeutic role of pharmacologic and endoscopic management in achieving hemostasis in NVUGIB, new modalities to improve the current treatment strategies continue to be developed. This review aims to identify some of the newer techniques as juxtaposed with the current therapeutic options.

\section{PHARMACOLOGICAL MANAGEMENT}

Various medications are used to either augment initial hemo- 
stasis or prevent the recurrence of bleeding during the same period of hospitalization. One of the stalwarts in this group are PPIs. It is known that an intragastric $\mathrm{pH}$ of 6 or lower can convert pepsinogen to pepsin, which can cause disaggregation of platelet-rich clots formed at the source of mucosal breakdown. ${ }^{3}$ On this basis, attempts to achieve gastric $\mathrm{pH}$ neutrality via pharmacologic intervention have been one of the foundations of initial management of GI bleeding. A review of 24 trials found that PPIs significantly reduced rebleeding, the need for repeat endoscopic therapy, and the requirement for surgery. Despite these advantages, the use of PPIs did not translate into a survival benefit. ${ }^{4}$ This could be attributed to the fact that PPIs are more useful in the maintenance of hemostasis rather than in initiating the hemostatic cascade. Hence, since most mortality related to upper GI hemorrhage occurs because of an inability to achieve initial hemostasis (either spontaneous or via endoscopic, angiographic, or surgical intervention), PPIs will not influence the mortality outcome in a statistically significant manner.

While PPIs certainly have their role in the armamentarium of NVUGIB, various dosing regimens and routes of administration (intravenous or oral) have been used. In fact, a Cochrane database review of 22 randomized controlled trials on various dosing regimens and routes (oral or intravenous) of administration found no difference with respect to rebleeding, repeat endoscopic therapy, need for emergency surgery, or mortality. ${ }^{5}$ Given this lack of clear benefit in prolonged IV administration and the substantially higher cost associated with intravenous PPIs, the current recommendation is to institute an oral PPI regimen as early as possible.

In contrast, there is much debate regarding the use of tranexamic acid (TXA; brand name, Lysteda; Ferring Pharmaceuticals Inc., St. Prex, Switzerland) in the management of acute NVUGIB. As a derivative of the amino acid lysine, it acts as an antifibrinolytic agent by inhibiting the action of plasmin. Owing to its role in the coagulation cascade, TXA has been used in circumstances requiring acute reduction of excessive blood loss from postpartum hemorrhage and also from iatrogenic causes such as bleeding after oral surgery. While some studies have suggested a mortality benefit when TXA is used in the management of upper GI, other studies performed since the advent of PPI therapy seem to provide a less robust degree of benefit, ultimately challenging its usefulness in the PPI era. ${ }^{6.7}$ Moreover, some concerns remain regarding the durability and consistency of its thrombogenic potential in acidic environments. Given the lack of high-quality evidence, the routine use of TXA is not currently recommended in general clinical practice. The hemorrhage alleviation with tranexamic acid-intestinal system (HALT-IT) trial, a large randomized, double-blind, placebo-controlled trial (Clinicaltrials.gov NCT01658124), is being conducted to clarify this issue by comparing patients administered TXA with those administered placebo, in order to quantify the effects of early administration of TXA on mortality, necessity for angiographic embolization and surgery, blood transfusion requirements, and risk of thromboembolic disease. ${ }^{8}$

\section{ENDOSCOPIC PHARMACOTHERAPY}

In general, endoscopic hemostasis relies either on the ablation of a bleeding vessel/lesion (thermal ablative method) or on mechanical tamponade of a bleeding source (see below). To improve the outcome of endoscopic hemostasis, various types of thermal ablative catheters and mechanical hemostatic tools (such as endoscopic hemoclips and multi-band ligators) were developed over the last three decades. This, in turn, resulted in an improved success rate of endoscopic hemostasis and a reduction in blood transfusion requirements. While proven to be effective and safe in managing most focal sources of NVUGIB, these methods are often inadequate when the bleeding source is atypical (such as tumor-related bleeding), multifocal, or diffuse.

With respect to endoscopically delivered pharmacotherapy, a number of topical hemostatic agents have been developed and incorporated into disposable endoscopic devices in recent years. The concept of topical application of hemostatic agents is attractive, as it is a non-traumatic method of hemostasis (i.e., no further destruction of the surrounding tissue during an attempt of hemostasis) that can be applied to a diffuse, multifocal source of bleeding. The significance of this type of hemostatic method is that endoscopic hemostasis can now reach beyond addressing single or focal sources of NVUGIB, such as peptic ulcer bleeding, towards the management of bleeding lesions that were not amenable to previous methods. ${ }^{9}$

One example of such methodology is the Hemospray. Hemospray (otherwise known as TC-325; Cook Medical, Bloomington, IN, USA) is an inorganic, non-absorbable nano-compound sprayed through a catheter that is inserted into the working channel of an endoscope (through-the-scope method). The powder is delivered with the aid of a pressurized $\mathrm{CO}_{2}$ canister to a desired location. The powder absorbs water, forming an adhesive barrier that creates a hemostatic plug. ${ }^{10}$ Small randomized and observational studies have demonstrated the efficacy and safety of Hemospray in managing bleeding from peptic ulcers as well as other iatrogenic etiologies. ${ }^{11}$ When Hemospray was used for various causes of NVUGIB, a review of all prospective and retrospective studies, and case series showed an immediate hemostasis of $92.3 \%$, with a 7 -day rebleeding rate of $20.6 \%{ }^{12}$ Given this proven shortterm benefit, some European, Asian, and American countries 
have approved the use of Hemospray as part of their arsenal of endoscopic hemostasis. However, in the United States, it is unavailable at present and is pending U S Food and Drug Administration (FDA) review.

The EndoClot (EndoClot Plus Inc., Santa Clara, CA, USA) polysaccharide hemostatic system is another non-contact endoscopic device that delivers hemostatic powder. As it is composed of absorbable modified polymer (AMP) particles, it absorbs water from blood. This dehydration process increases the concentration of platelets, red blood cells, and coagulation proteins (thrombin, fibrinogen, etc.), resulting in the rapid formation of a hemostatic layer. This device primarily originated outside of the United States, and multiple small studies have demonstrated its potential efficacy and safety in managing NVUGIB. ${ }^{13,14}$

Ankaferd Blood Stopper (ABS; Ankaferd Health Products, Istanbul, Turkey) is a $100-\mathrm{mL}$ herbal extract composed of five different plant species, namely $9 \mathrm{mg}$ of Glycyrrhiza glabra (dried leaf extract), $5 \mathrm{mg}$ of Thymus vulgaris (dried grass extract), $7 \mathrm{mg}$ of Alpinia officinarum (dried leaf extract), $6 \mathrm{mg}$ of Urtica dioica (dried root extract), and $8 \mathrm{mg}$ of Vitis vinifera (dried leaf extract). The exact mechanism of action of ABS is unknown, but it is purported to form an encapsulated web of erythrocyte aggregation. While ABS has not been approved by the FDA, one retrospective observational series of $26 \mathrm{pa}-$ tients showed that its application via a disposable wash pipe was effective in all cases except spurting arterial bleeding. ${ }^{15}$

It is important to iterate that all aforementioned hemostatic compounds are at best a temporary remedy, providing shortterm hemostasis, and that long-term hemostasis relies on mucosal healing under the hemostatic plug created by these various compounds.

\section{TECHNIQUES OF ENDOSCOPIC HEMOSTASIS}

Conventional therapy for treatment of various forms of NVUGIB includes injectants (specifically epinephrine to achieve tamponade), mechanical therapy (specifically endoscopic clips), and cautery (including heater, monopolar, and bipolar electrocautery), which cause coaptation, and argon plasma coagulation, which causes coagulation in the absence of mechanical contact. ${ }^{16}$ It is imperative to recognize that monotherapy, which relies only on the injection of a vasoconstrictive substance, is inadequate in achieving a meaningful long-term hemostasis, with an unacceptable risk of recurrent bleeding and a significantly higher rate of requirement of surgical intervention. On comparing these various modalities, specifically with respect to ulcer bleeding, thermal therapy or clips are found to outperform epinephrine monotherapy, whereas epinephrine plus an additional modality outperform epinephrine monotherapy. Thermal ablative methods and mechanical hemostasis as monotherapy have resulted in a largely comparable rate of successful endoscopic hemostasis. ${ }^{17}$

New technologies have been developed to address and optimize the treatment of NVUGIB with respect to mechanical endoscopic hemostasis. These include over-the-scope clips (OTSCs). The OTSC, otherwise known as the "bear claw," comes in various dimensions with blunt or sharp closure jaws. Similar in deployment to the variceal banding kit, the opposing ends of a bleeding lesion are suctioned into the endoscopic apparatus with subsequent deployment of the device. While prospective randomized clinical trials have yet to be performed, the largest case series in the literature to date included 30 consecutive patients with either upper or lower GI bleeding refractory to conventional endoscopic techniques (defined as epinephrine injection and throughthe-scope clips). Initial hemostasis was achieved in $97 \%$ of the patients, with one patient requiring interventional radiology embolization from a duodenal bulb ulcer. Rebleeding occurred in two patients who were amenable to conventional techniques. These results were, thus, in favor of OTSC as a useful modality when conventional hemostatic therapy has failed. $^{18}$

Another technology that is gaining acceptance is radiofrequency ablation (RFA), particularly in the setting of gastric antral vascular ectasia (GAVE). Using uniform thermal energy in a superficial manner, RFA has been used to treat GAVE refractory to argon plasma coagulation. In an open-label prospective cohort study, 21 patients with transfusion-dependent GAVE underwent RFA, with 18 of them achieving transfusion independence. ${ }^{19}$ Similarly, in an open-label, retrospective case series, 24 patients with GAVE underwent RFA, and 15 became transfusion independent at 6 months. ${ }^{20}$

Cryotherapy, a modality that uses compressed carbon dioxide or liquid nitrogen to freeze tissues and cause superficial necrosis, has also been used in patients with refractory GAVE. In one pilot study that assessed 12 patients with GAVE and anemia, 50\% of the patients achieved a complete response, while the rest achieved a partial response with decreased transfusion requirements. However, the best delivery system and the number of treatments required still remain unclear. $^{21}$

\section{CONCLUSIONS}

In summary, several pharmacologic and endoscopic modal- 
ities exist in the endoscopist's armamentarium for NVUGIB. Targeted pharmacological and endoscopic therapies include epinephrine as well as endoscopic ablative treatment and mechanical tamponade. Multifocal lesions that are difficult to control with targeted therapy have prompted a burgeoning of hemostatic powder delivery systems that afford the endoscopist the ability to achieve hemostatic control when the location and/or number of lesions render the targeted therapy less advantageous. While some modalities have not been approved by the FDA at this juncture, improved technique, device design, and pharmacologic adjuncts will enhance outcomes with respect to hemostatic control achieved by the index endoscopic intervention.

\section{Conflicts of Interest}

The authors have no financial conflicts of interest.

\section{REFERENCES}

1. Tielleman T, Bujanda D, Cryer B. Epidemiology and risk factors for upper gastrointestinal bleeding. Gastrointest Endosc Clin N Am 2015;25:415-428.

2. Abougergi MS, Travis AC, Saltzman JR. The in-hospital mortality rate for upper GI hemorrhage has decreased over 2 decades in the United States: a nationwide analysis. Gastrointest Endosc 2015;81:882-888.e1.

3. Lam KL, Wong JC, Lau JY. Pharmacological treatment in upper gastrointestinal bleeding. Curr Treat Options Gastroenterol 2015;13:369-376.

4. Leontiadis GI, Sharma VK, Howden CW. Proton pump inhibitor therapy for peptic ulcer bleeding: Cochrane collaboration meta-analysis of randomized controlled trials. Mayo Clin Proc 2007;82:286-296.

5. Neumann I, Letelier LM, Rada G, et al. Comparison of different regimens of proton pump inhibitors for acute peptic ulcer bleeding. Cochrane Database Syst Rev 2013;(6):CD007999.

6. Bennett C, Klingenberg SL, Langholz E, Gluud LL. Tranexamic acid for upper gastrointestinal bleeding. Cochrane Database Syst Rev 2014;(11): CD006640.

7. Fortinsky KJ, Bardou M, Barkun AN. Role of medical therapy for non- variceal upper gastrointestinal bleeding. Gastrointest Endosc Clin N Am 2015;25:463-478.

8. Roberts I, Coats T, Edwards P, et al. HALT-IT: tranexamic acid for the treatment of gastrointestinal bleeding: study protocol for a randomised controlled trial. Trials 2014;15:450.

9. Bergel S. The effect of the fibrin. Dtsch Med Wochenschr 1909;35:663-5.

10. Hemospray. A different approach to hemostasis [Internet]. Bloomington: Cook Medical; c2016 [cited 2016 Sep 13]. Available from: http:// hemospray.cookmedical.com/region-two/hemostasis-treatment.html.

11. Yau AH, Ou G, Galorport C, et al. Safety and efficacy of Hemospray(R) in upper gastrointestinal bleeding. Can J Gastroenterol Hepatol 2014;28:7276.

12. Chen YI, Barkun AN. Hemostatic powders in gastrointestinal bleeding a systematic review. Gastrointest Endosc Clin N Am 2015;25:535-552.

13. Müller-Cerbes D, Beeck A, Dormann A, et al. Hemostasis with powder: experience with EndoClot in difficult Upper GI bleedings. Endosk heute $2013 ; 26: 254-258$

14. Halkerston K, Evans J, Ismail D, et al. Early clinical experience of Endoclot in the treatment of acute gastro-intestinal bleeding. Gut 2013;62(Suppl 1):A149.

15. Kurt M, Onal I, Akdogan M, et al. Ankaferd blood stopper for controlling gastrointestinal bleeding due to distinct benign lesions refractory to conventional antihemorrhagic measures. Can J Gastroenterol 2010;24:380-384

16. Hwang JH, Fisher DA, Ben-Menachem T, et al. The role of endoscopy in the management of acute non-variceal upper GI bleeding. Gastrointest Endosc 2012;75:1132-1138.

17. Laine L, McQuaid KR. Endoscopic therapy for bleeding ulcers: an evidence-based approach based on meta-analyses of randomized controlled trials. Clin Gastroenterol Hepatol 2009;7:33-47.

18. Manta R, Galloro G, Mangiavillano B, et al. Over-the-scope clip (OTSC) represents an effective endoscopic treatment for acute GI bleeding after failure of conventional techniques. Surg Endosc 2013;27:3162-3164.

19. McGorisk T, Krishnan K, Keefer L, Komanduri S. Radiofrequency ablation for refractory gastric antral vascular ectasia (with video). Gastrointest Endosc 2013;78:584-588.

20. Dray X, Repici A, Gonzalez P, et al. Radiofrequency ablation for the treatment of gastric antral vascular ectasia. Endoscopy 2014;46:963-969.

21. Cho S, Zanati S, Yong E, et al. Endoscopic cryotherapy for the management of gastric antral vascular ectasia. Gastrointest Endosc 2008;68:895902. 